\title{
Completing Artin's braid group on infinitely many strands
}

\author{
Paul Fabel \\ Department of Mathematics \& Statistics \\ Mississippi State University
}

\begin{abstract}
A generalization of the topological fundamental group is developed in order to construct a completion of Artin's braid group on infinitely many strands with respect to the following notion of convergence: $b_{n} \rightarrow i d$ iff for each $M>0$, eventually the first $M$ strands of $b_{n}$ are trivial.
\end{abstract}

\section{Introduction}

Artin's braid group $B_{n}$ is the fundamental group of the collection of planar sets each of which has $n$ elements. Fixing a basepoint $Z_{n} \subset R^{2}$, elements of $B_{n}$ can be seen as collections of disjoint arcs in $R^{2} \times[0,1]$ connecting $Z_{n} \times\{0\}$ to $Z_{n} \times\{1\}$.

Artin's braid group on infinitely many strands $B_{\infty}$ is the direct limit of $B_{n}$ under monomorphisms $i_{n}: B_{n} \rightarrow B_{n+1}$ that attach a "trivial strand'. It has been studied from various perspectives by Broto [2], Dehornoy [3] [4, Dynnikov [5], Moran [8], Kim and Rolfsen [10, Vershinin [11] and others.

The group $B_{\infty}$ becomes a topological group under the requirement that a sequence of braids $b_{n} \rightarrow i d \in B_{\infty}$ iff for each $M$ there exists $N$ such that if $n \geq N$ then the first $M$ strands of $b_{n}$ are trivial.

Unfortunately $B_{\infty}$ admits no complete metric compatible with its topology. The goal of this paper is to enlarge $B_{\infty}$ to a complete group of braids on infinitely many strands.

Recent work of Biss [1] shows generally that the based fundamental group of a space $\pi_{1}(X, p)$ inherits a canonical topology from $X$ and becomes a topological group.

In an effort order to complete $B_{\infty}$, we develop a generalization of the topological fundamental group and subsequently exhibit a completely 
metrizable topological group $\overline{B_{\infty}}$ such that $B_{\infty}$ is embedded in $\overline{B_{\infty}}$ as a dense subgroup. By construction each element of $\overline{B_{\infty}}$ can be seen as a braid with infinitely many strands, a countable collection of pairwise disjoint arcs in $R^{2} \times[0,1]$.

The bulk of this paper is devoted to proving, for a certain space $X$ ( the space of injections of the integers into the plane), that the topological fundamental group of $X$ is isomorphic to $\lim _{\leftarrow} G_{n}$, the inverse limit of Artin's pure braid groups on $n$ strands. This serves to complete Artin's pure braid group on infinitely many strands, the subgroup of braids where each strand has identical endpoints. A more general construction is then employed to handle complications created by permutation of the strand endpoints.

The construction of $\overline{B_{\infty}}$ in this paper serves as a counterpart to that of [6] in which $B_{\infty}$ is completed into a group of tame braids via the mapping class group of a disk with infinitely many punctures. However the topology of $B_{\infty}$ in 6 ] is finer than that of the current paper. In [6] a sequence of braids $b_{n}$ converges to $i d$ iff the first $M$ strands are eventually trivial and unlinked from the remaining strands. The latter notion of convergence in $B_{\infty}$ is compatible with a left order topology of Dehornoy [3, and the construction in [6] seems to resolve the issue of its completion as suggested by Dehornoy in [4].

By contrast, with the coarser topology of $B_{\infty}$ adopted in the current paper, some elements of $\overline{B_{\infty}}$ are 'wild' in a strong sense: each representative of a wild element is not the restriction of an ambient isotopy of the plane.

\section{Preliminaries}

Spaces of continuous functions will have the compact open topology, product spaces will have the product topology, and quotient spaces will have the quotient topology.

Given topological spaces $A$ and $Y$ let $C(A, Y)$ denote the continuous functions from $A$ into $Y$. Let $I(A, Y)=\{f \in C(A, Y) \mid f$ is one to one $\}$. Note elements of $I(A, Y)$ are not necessarily embeddings. Let $E(A, Y)=\{f \in C(A, Y) \mid f$ is a surjection $\}$. Let $H(A, A)$ denote the group of homeomorphisms from $A$ onto $A$.

A surjective map $q: A \rightarrow Y$ is a quotient map provided $V$ is open in $Y$ if and only if $q^{-1}(V)$ is open in $A$. The following propositions (Theorem 11.1 p. 139 and Corollary 5.4 p287) are proved in Munkres [9].

Proposition 1 If $q: A \rightarrow Y$ is a quotient map and $f: A \rightarrow B$ a map such that $f$ is constant on sets of the form $q^{-1}(y)$ then there exists a 
map $F: Y \rightarrow B$ such that $f=F(q)$.

Proposition 2 If $X$ is locally compact and Hausdorf then $F: X \times Z \rightarrow$ $Y$ is continuous iff $F: Z \rightarrow C(X, Y)$ is continuous where $F$ is defined by $(F(z))(x)=F(x, z)$.

If $A_{1}, A_{2}, \ldots$ is a sequence of sets and $\psi_{n}: A_{n} \rightarrow A_{n-1}$ is a surjection then the inverse limit of $A_{n}$ under $\psi_{n}$ is the denoted $\lim _{\leftarrow} A_{n}$ and consists of all sequences $\left(a_{1}, a_{2}, \ldots\right)$ such that $a_{n} \in A_{n}$ and $\psi_{n}\left(a_{n}\right)=a_{n-1}$.

If the topological space $G$ is a group with multiplication $m$ and inversion $v$ then $G$ is a topological group provided $m$ and $v$ are continuous.

Let $Z^{+}=\left\{(n, 0) \in R^{2} \mid n \in\{1,2,3, .\}.\right\}$.

Let $Z_{n}=\left\{(k, 0) \in Z^{+} \mid 1 \leq k \leq n\right\}$.

Lemma 3 The map $\pi: H\left(R^{2}, R^{2}\right) \rightarrow I\left(Z_{n}, R^{2}\right)$ defined via $\pi(h)=h_{\mid Z_{n}}$ determines a fibre bundle.

Proof. Given $x$ in the interior of a closed round planar disk $D$ there is a circle's worth of line segments each of which has one endpoint $x$ and the other endpoint on $\partial D$. Given $\{x, y\} \subset \operatorname{int}(D)$ there is a canonical homeomorphism $h(D, x, y): D \rightarrow D$ mapping $x$ to $y$, mapping one segment linearly onto another, and fixing pointwise $\partial D$. Given $\alpha \in$ $I\left(Z_{n}, R^{2}\right)$ choose $h_{\alpha}$ such that $\pi\left(h_{\alpha}\right)=\alpha=h_{\alpha \mid Z_{n}}$. Choose disjoint closed disks $D_{i}$ centered at $\alpha(i)$. For $\beta$ close to $\alpha$ let $h_{\alpha, \beta}: R^{2} \rightarrow R^{2}$ map $\alpha(i)$ to $\beta(i)$, leaving invariant $D_{i}$ via the canonical homeomorphism, and fixing pointwise $R^{2} \backslash \cup D_{i}$. Let $F=\left\{h \in H\left(R^{2}, R^{2}\right) \mid h_{\mid Z_{n}}=i d_{\mid Z_{n}}\right\}$. For a small neighborhood $U$ of $\alpha$ define $\phi: U \times F \rightarrow H\left(R^{2}, R^{2}\right)$ by $\phi(\beta, f)=\left(h_{\alpha \beta}\right) h_{\alpha} f$.

Lemma 4 Suppose $\left[\alpha_{0}\right]=[i d] \in \pi_{1}\left(I\left(Z_{n}, R^{2}\right), i d_{\mid Z_{n}}\right)$ and $\alpha_{t}$ is a path homotopy from $\alpha_{0}$ to id in $I\left(Z_{n}, R^{2}\right)$. Then there exists a level preserving isotopy $h_{t}: R^{2} \times[0,1] \rightarrow R^{2} \times[0,1]$ such that $h_{t \mid Z_{n}}=\alpha_{t}, h_{1}=i d_{\mid R^{2} \times[0,1]}$, and $h_{t}$ is the identity on $R^{2} \times\{0\}$ and $R^{2} \times\{1\}$.

Proof. Define a map $q:[0,1] \times[0,1] \rightarrow I(F, \operatorname{int}(D))$ via $q(s, t)=$ $\alpha_{t}(s)$. Note $i d_{\mid F}=q(0, t)=q(s, 1)=q(1, t)$. The space obtained by collapsing 3 consecutive sides of $[0,1] \times[0,1]$ to a point is a topologically a disk. Thus by Proposition $3 q$ lifts to a map $Q:[0,1] \times[0,1] \rightarrow H\left(R^{2}, R^{2}\right)$ such that $\pi(Q)=q$ and $i d_{\mid D}=Q(0, t)=Q(s, 1)=Q(1, t)$. Define $h_{t}: R^{2} \times[0,1] \rightarrow R^{2} \times[0,1]$ via $h_{t}(z, s)=Q(s, t)(z)$.

Lemma 5 If $Z=\{1,2,3, \ldots\}$ then $H(Z, Z)$ is a completely metrizable topological group. 
Proof. For $\{n, m\} \subset Z$ let $d^{*}(n, m)=1$ if $n \neq m$ and $d^{*}(n, m)=$ 0 if $n=m$. Note $C(Z, Z)$ is complete under the metric $d(f, g)=$ $\sum_{n=1}^{\infty} \frac{d^{*}(f(n), g(n))}{2^{n}}$. Let $E^{n} \subset C(Z, Z)$ denote the maps whose image contains $\{1, \ldots n\}$. Suppose $f \in E^{n}$. Choose finite $M \subset Z$ such that $\{1, . . n\} \subset f(M)$ and $k=f\left(m_{k}\right)$ with $1 \leq k \leq n$ and $m_{k} \in M$. Note for $g$ sufficiently close to $f$ we have $g\left(m_{k}\right)=f\left(m_{k}\right)$. Hence $E^{n}$ is open in $C(Z, Z)$. Thus $E(Z, Z)$ is a $G_{\delta}$ subspace of $C(Z, Z)$ since $E(Z, Z)=\cap E^{n}$. In particular $E(Z, Z)$ is completely metrizable (p.270 [9]). Let $I^{n} \subset C(Z, Z)$ denote the maps $f$ such that $f_{\{\{1, . ., n\}}$ is one to one. Suppose $f \notin I^{n}$. Then for $g$ sufficiently close to $f$ we have $f_{\mid\{1, ., n\}}=g_{\mid\{1, . ., n\}}$. Hence $I^{n}$ is closed in $C(Z, Z)$. Thus $I(Z, Z)$ is closed in $C(Z, Z)$ since $I(Z, Z)=\cap I_{n}$. Note $H(Z, Z)=I(Z, Z) \cap E(Z, Z)$. Hence $H(Z, Z)$ is completely metrizable since it's a closed subspace of the completely metrizable space $E(Z, Z)$.

To prove $H(Z, Z)$ is a topological group note first that compact sets in $Z$ are exactly the finite subsets of $Z$. Hence convergence in $H(Z, Z)$ is equivalent to pointwise convergence. If $f_{n} \rightarrow f$ and $g_{n} \rightarrow g$ then $\lim _{n \rightarrow \infty} f_{n}\left(g_{n}\right)(m) \rightarrow f(g)(m)$. Thus function composition is continuous in $H(Z, Z)$. To check inversion is continuous it suffices to check the case when $h_{n} \rightarrow i d$. Fixing $i$ choose $N$ such that $h_{n}(i)=i$ whenever $n \geq N$. In particular $h_{n}^{-1}(i)=i$. Thus $h_{n}^{-1} \rightarrow i d$, and hence $H(Z, Z)$ is a topological group.

Lemma $6 B_{\infty}$ is topologically incomplete with the topology described in the introduction.

Proof. Note that $B_{\infty}$ is countable and that the complement of every one point subset of $B_{\infty}$ is open and dense. Thus $B_{\infty}$ is the countable union of closed nowhere dense sets. Hence by the Baire category theorem $B_{\infty}$ does not admit a complete metric.

\subsection{Generalizing the topological fundamental group}

It is unknown whether there exists a space whose topological fundamental group completes $B_{\infty}$ with the topology described in the introduction. To bypass this difficulty we develop a generalization of the topological fundamental group. The basic idea is to replace the basepoint of $X$ with a subset $A \subset X$, and to consider paths of embeddings of $A$ into $X$ that initially fix $A$ pointwise, that are allowed to move $A$ off of itself in midjourney, and that place $A$ back onto a permutation of itself at the last moment. Under suitably nice conditions the path homotopy classes determine a topological group.

Suppose throughout this section that $A \subset Y,\left\{i d_{A}\right\} \subset X \subset I(A, Y)$, and $H$ is a subgroup of $H(A, A)$. 
Let $K=\left\{f \in C([0,1], X) \mid f(0)=i d_{A}, f(1) \in H\right\}$.

Define $m: K \times K \rightarrow K$ such that

$$
m(f, g)(t)=\left\{\begin{array}{cc}
f(2 t) & t \leq 1 / 2 \\
g(2 t-1) f(1) & 1 / 2 \leq 1
\end{array}\right.
$$

Define $v: K \rightarrow K$ such that $(v f)(t)=f(1-t)(f(1))^{-1}$ whenever $f \in K$.

Endowed with the quotient topology, let $G(X, H)$ denote the space whose elements are path components of $K$.

Theorem 7 If $H$ is a topological group then $m$ and $v$ induce a topological group structure on $G(X, H)$.

Proof. Evidently $m$ is continuous and $v$ is continuous since inversion in $H$ is continuous. Given $f \in K$, let $[f]$ denote the path component of $f$ in $K$. Let $q: K \rightarrow G(X, H)$ be the quotient map $q(f)=[f]$. Define $M: G(X, H) \times G(X, H) \rightarrow G(X, H)$ such that $M([f],[g])=[m(f, g)]$. Define $V: G(X, H) \rightarrow G(X, H)$ via $V([f])=[v(f)]$. To check that $M$ and $V$ are well defined and continuous it suffices by Proposition 1 to note that $q(m)$ and $q(j)$ are constant respectively on sets $(q \times q)^{-1}([f],[g])$ and $q^{-1}([f])$. Define $I D:[0,1] \rightarrow K$ via $I D(t)=i d_{A}$. The proof that $G(X, H)$ is a group with identity $[I D]$, multiplication $M$, and inversion $V$ is similar to the proof that the familiar fundamental group is indeed a group. Note the paths $m(I D, f), m(f, I D)$ and $f$ all differ by an order preserving surjective map of $[0,1]$ and hence $M([I D],[f])=$ $M([I D],[f])=[f]$. Similarly the maps $m(m(f, g), h)$ and $m(f, m(g, h))$ differ by an order preserving surjective map of $[0,1]$ and thus $M$ is associative. Note $m(f, v(f))=m(v(f), f)$. The path $m(f, v(f))$ can be deformed to $I D$ by concatenating $f_{[s, 0]}$ with $f_{[s, 0]}$. Thus $V$ serves as inversion and hence $G(X, H)$ is a topological group.

The based topological fundamental group $\pi_{1}(Y, p)$ [1] is the quotient space of loops based at $p$ from $[0,1]$ into $Y$ under the equivalence relation path homotopy. This can be seen as a special case of the generalized topological fundamental group $G(X, H)$.

Corollary 8 If $A=\{p\} \subset Y, H=\left\{i d_{A}\right\}$ and $X=I(\{p\}, Y)$ then $G(X, H) \cong \pi_{1}(Y, p)$, the topological fundamental group of $X$ based at $p$.

\subsection{Twisted Products and Inverse Limit Spaces}

Lemma 12 helps establish that the inverse limit of the groups $G_{n}$ is canonically isomorphic (and homeomorphic) to an iterated semidirect product of groups $K_{n}$. 
Definition 9 Suppose each of $G_{1}, G_{2}, . . K_{1}, K_{2}, .$. is a group such that for all $n \geq 1 \cup_{i=1}^{n} K_{i} \subset G_{n}$ and $K_{n} \triangleleft G_{n}$. Then $\prod_{n=1}^{\infty} K_{n}$ forms a group under the binary operation determined coordinatewise by

$$
\left[\left(x_{1}, x_{2}, . .\right) *\left(y_{1}, y_{2}, \ldots\right)\right]_{n}=\left(y_{1} \ldots y_{n-1}\right)^{-1}\left(x_{n}\right)\left(y_{1} . . y_{n-1}\right) y_{n}
$$

(with multiplication performed in $G_{n}$ ). Call this group $\Pi_{G_{n}} K_{n}$, the twisted product of $K_{n}$ over $G_{n}$.

Lemma 10 The twisted product is a well defined group.

Proof. Note $*$ is well defined since $K_{n} \triangleleft G_{n}$ and $K_{i} \subset G_{n}$ for $1 \leq i<n$. Checking associativity,

$$
\begin{gathered}
{[x *(y * z)]_{n}=\left[\left(y_{1} z_{1}\right)\left(z_{1}^{-1} y_{2} z_{1} z_{2}\right)\left(\left(z_{1} z_{2}\right)^{-1} y_{3}\left(z_{1} z_{2} z_{3}\right)\right) \ldots\right.} \\
\left.\left(\left(z_{1} \ldots z_{n-2}\right)^{-1} y_{n-1} z_{1} z_{2} \ldots z_{n-1}\right)\right]^{-1} x_{n}\left[\left(y_{1} z_{1}\right)\left(z_{1}^{-1} y_{2} z_{1} z_{2}\right)\left(\left(z_{1} z_{2}\right)^{-1} y_{3}\left(z_{1} z_{2} z_{3}\right)\right) \ldots\right. \\
\left.\left(\left(z_{1} \ldots z_{n-2}\right)^{-1} y_{n-1} z_{1} z_{2} \ldots z_{n-1}\right)\right]\left(z_{1} \ldots z_{n-1}\right)^{-1} y_{n}\left(z_{1} \ldots z_{n}\right)= \\
\left(z_{1} . . z_{n-1}\right)^{-1}\left(y_{1} \ldots y_{n-1}\right)^{-1} x_{n}\left(y_{1} . . y_{n}\right)\left(z_{1} . . z_{n}\right)=[(x * y) * z]_{n} .
\end{gathered}
$$

Let $I D=(i d, i d, .$.$) . Then [I D x]_{n}=x_{n}=[x I D]_{n}$. Finally given $y=\left(y_{1}, y_{2}, ..\right)$ let $x=\left(x_{1}, x_{2}, ..\right)$ with $x_{n}=\left(y_{1} \ldots y_{n-1}\right)\left(y_{1} \ldots y_{n-1} y_{n}\right)^{-1}$. Then

$$
[x * y]_{n}=\left(y_{1} . . y_{n-1}\right)^{-1}\left(y_{1} \ldots y_{n-1}\right)\left(y_{n}\right)^{-1}\left(y_{1} \ldots y_{n-1}\right)^{-1}\left(y_{1} \ldots y_{n}\right)=i d .
$$

Lemma 11 Suppose $G$ is a group with subgroups $\{i d\}=G_{0} \subset G_{1} \subset$ $G_{2} \subset G_{3} \ldots \subset G$ and there exist homomorphisms $\phi_{n}: G \rightarrow G_{n}$ for all $n \geq 0$ satisfying $\phi_{n \mid G_{n}}=i d_{\mid G_{n}}$. Define for all $n \geq 1 \psi_{n}: G_{n} \rightarrow G_{n-1}$ as $\psi_{n}=\phi_{n-1 \mid G_{n}}$. Let $K_{n}=\operatorname{ker} \psi_{n}$. Then $\phi: G \rightarrow \Pi_{G_{n}} K_{i}$ defined as $(\phi(g))_{n}=\left(\phi_{n-1}(g)\right)^{-1} \phi_{n}(g)$ is a homomorphism.

Proof. Note $K_{n}=\operatorname{ker} \psi_{n} \triangleleft G_{n}$ and $K_{i} \subset G_{i} \subset G_{n}$ for $1 \leq i<$ $n$ and the hypothesis of Definition 9 is satisfied. To check $i m(\phi) \subset$ $\Pi K_{i}$ we verify $\left(\phi_{n-1}(g)\right)^{-1} \phi_{n}(g) \in K_{n}$. Let $p=\phi_{n}(g)$. Note $\phi_{n-1}(g)=$ $\phi_{n-1}\left(\phi_{n}(g)\right)=\phi_{n-1}(p)$ and $\phi_{n-1}\left(\phi_{n-1}(x)\right)=\phi_{n-1}(x)$ for all $x \in G$. Thus $\psi_{n}\left(\left(\phi_{n-1}(p)\right)^{-1} p\right)=\phi_{n-1}\left(\phi_{n-1}\left(p^{-1}\right)\right) \phi_{n-1}(p)=\left(\phi_{n-1}\left(p^{-1}\right)\right) \phi_{n-1}(p)=i d$.

To check $\phi$ is a homomorphism use the following notation. For $g \in G$ let $g_{n}=(\phi(g))_{n} \in K_{n}$ and let $g^{(n)}=\phi_{n}(g) \in G_{n}$. Note

$$
g^{(n)}=\left(g^{(0)}\right)^{-1}\left(g^{(1)}\right)\left(g^{(1)}\right)^{-1} . .\left(g^{(n-1)}\right)^{-1} g^{(n)}=g_{1} g_{2} \ldots g_{n} .
$$


Thus

$$
\begin{gathered}
(\phi(g h))_{n}=\left(\phi_{n-1}(g h)\right)^{-1} \phi_{n}(g h)=\phi_{n-1}\left(h^{-1}\right)\left(\phi_{n-1}\left(g^{-1}\right) \phi_{n}(g) \phi_{n}(h)=\right. \\
\left(h^{(n-1)}\right)^{-1} g_{n} h^{(n)}=\left(h_{1} . . h_{n-1}\right) g_{n}\left(h_{1} \ldots h_{n-1}\right) h_{n}=(\phi(g) * \phi(h))_{n} .
\end{gathered}
$$

Lemma 12 Suppose $\{i d\}=G_{0} \subset G_{1} \subset G_{2}$.. is a sequence of groups and $\psi_{n}: G_{n} \rightarrow G_{n-1}$ is an epimorphism such that $\psi_{n \mid G_{n-1}}=i d_{\mid G_{n-1}}$. Let $\lim _{\leftarrow} G_{n}=\left\{\left(g_{1}, g_{2}, ..\right) \mid g_{n} \in G_{n}\right.$ and $\left.\psi_{n}\left(g_{n}\right)=g_{n-1}\right\}$ with group operation $\left(g_{1}, g_{2}, ..\right) *\left(h_{1}, h_{2}, ..\right)=\left(g_{1} h_{1}, g_{2} h_{2}, ..\right)$. Let $K_{n}=\operatorname{ker} \psi_{n}$. Then $\psi: \Pi_{G_{n}} K_{n} \rightarrow \lim _{\leftarrow} G_{n}$ defined as $\psi\left(k_{1}, k_{2}, ..\right)=\left(k_{1}, k_{1} k_{2}, ..\right)$ is an isomorphism. If for all $n G_{n}$ has the discrete topology and $\lim _{\leftarrow} G_{n}$ and $\Pi_{G_{n}} K_{n}$ have the product topology then $\psi$ is a homeomorphism.

Proof. To check $\psi$ is a homomorphism

$$
\begin{gathered}
\psi\left[\left(x_{1}, x_{2}, . .\right)\left(y_{1}, y_{2}, . .\right)\right]=\phi\left(x_{1} y_{1},\left(y_{1}\right)^{-1} x_{2} y_{1} y_{2}, \ldots\right)= \\
x_{1} y_{1}, x_{1} x_{2} y_{1} y_{2}, . .=\left(x_{1}, x_{1} x_{2}, . .\right) *\left(y_{1}, y_{1} y_{2}, . .\right)=\psi\left(x_{1}, x_{2}, . .\right) * \psi\left(y_{1}, y_{2}, . .\right) .
\end{gathered}
$$

Suppose $\psi\left(k_{1}, k_{2}, ..\right)=(i d, i d, .$.$) . Then k_{1}=i d, k_{1} k_{2}=i d, \ldots$ Hence by induction $k_{n}=i d$ for all $n$. Suppose $\left(g_{1}, g_{2}, ..\right) \in \lim _{\leftarrow} G_{n}$. Let $k_{1}=g_{1}$. Suppose by induction that $g_{n-1}=k_{1} \ldots k_{n-1}$ with $k_{i} \in K_{i}$. Let $k_{n}=$ $\left(g_{n-1}\right)^{-1} g_{n}$.

Note

$$
\psi_{n}\left(k_{n}\right)=\psi_{n}\left(\left(g_{n-1}\right)^{-1} \psi_{n}\left(g_{n}\right)=g_{n-1}^{-1} \psi\left(g_{n}\right)=\psi\left(g_{n}^{-1}\right) \psi\left(g_{n}\right)=i d .\right.
$$

Thus $\psi$ is an isomorphism. Note $\lim _{\leftarrow} G_{n}$ and $\Pi_{G_{n}} K_{n}$ are metrizable since each of $G_{n}$ and $K_{n}$ is metrizable. Thus it suffices to prove the homomorphisms $\psi$ and $\psi^{-1}$ preserve convergence at $(i d, i d, .$.$) . Suppose$

$$
\left(k_{1}^{1}, k_{2}^{1}, k_{3}^{1}, \ldots\right),\left(k_{1}^{2}, k_{2}^{2}, k_{3}^{2}, . .\right), \ldots \rightarrow(i d, i d, i d, . .) .
$$

Suppose $N>0$. Choose $M$ so that $k_{j}^{i}=i d$ for $i \geq M$ and $j \leq N$. Suppose $i \geq M$ then $\left[\psi\left(k_{1}^{i}, k_{2}^{i}, . .\right)\right]_{n}=i d$. Thus $\psi$ is continuous. Conversely suppose

$$
\left(g_{1}^{1}, g_{2}^{1}, g_{3}^{1}, . .\right),\left(g_{1}^{2}, g_{2}^{2}, g_{3}^{2}, . .\right), \ldots \rightarrow(i d, i d, i d, \ldots) .
$$

Suppose $N>0$. Choose $M$ so that $i \geq M$ and $g_{N}^{i}=g_{N-1}^{i}=i d$. Then $k_{n}=i d$. Thus $\psi^{-1}$ is continuous. 


\section{The complete braid groups $G$ and $\overline{B_{\infty}}$ on infinitely many strands}

Let $X=I\left(Z^{+}, R^{2}\right)$

Let $X_{n}=\{f \in X \mid f(i, 0)=(i, 0)$ for all $i>n$ and for all $i \leq n$ $f(i, 0) \in(-\infty, n+2 / 3] \times R\}$.

Recalling Theorem [7, let $G=G\left(X,\left\{i d_{Z^{+}}\right\}\right)$, the topological fundamental group of $X$ based at $i d_{Z^{+}}$.

Viewed as a collection of arcs in $R^{2} \times[0,1]$, given $g \in[g] \in G$, the strands of $g$ connect $(n, 0,0)$ to $(n, 0,1)$ for $n \in\{1,2,3, \ldots\}$.

The main goal is to prove that $G$ is isomorphic to the inverse $\lim _{\leftarrow} G_{n}$ where $G_{n}$ is the pure braid group on $n$ strands, realized as the image of $G$ under retractions that move the first $n$ strands 'out of the way' and then replace the remaining strands with trivial strands.

\subsection{The retraction $P_{n}: X \rightarrow X_{n}$}

Lemma 13 There exists a retraction $P_{n}: X \rightarrow X_{n}$.

Proof. Define $R_{n}: X \rightarrow[0, \infty)$ as $R_{n}(g)=\min \{r \mid r \geq 0$ and for all $i \leq n g(i, 0) \in(-\infty, n+2 / 3+r] \times R\}$. Define $T: R \rightarrow C\left(R^{2}, R^{2}\right)$ as $T(r)(x, y)=(x-r, y)$. Define $P_{n}: X \rightarrow X_{n}$ via $P_{n}(g)(i, 0)=$ $\left\{\begin{array}{cl}\left(T R_{n} g\right)(g(i, 0)) & i \leq n \\ (i, 0) & i>n\end{array}\right.$.

\subsection{The groups $G_{n}$ and homomorphisms $\phi_{n}$ and $\phi$}

Suppose $n \geq 1$. Define $P_{n}^{*}: \pi_{1}(X, i d) \rightarrow \pi_{1}\left(X_{n}, i d\right)$ as $P_{n}^{*}[g]=\left[P_{n}(g)\right]$ where $P_{n}: X \rightarrow X_{n}$ is the retraction from Lemma [13] Let $i_{n}^{*}$ : $\pi_{1}\left(X_{n}, i d\right) \rightarrow \pi_{1}(X, i d)$ satisfy $i_{n}^{*}[\alpha]=[\alpha]$. Recall $G=\pi_{1}\left(X, i d_{Z^{+}}\right)$. Let $G_{n}=i m\left(i_{n}^{*}\right) \subset G$. Define $\phi_{n}: G \rightarrow G_{n}$ as $\phi_{n}=i_{n}^{*} P_{n}^{*}$. Let $G_{0}=\{[i d]\} \subset G$ and define $\phi_{0}: G \rightarrow G_{0}$. Let $K_{n}=G_{n} \cap \operatorname{ker} \phi_{n-1}$. Define $\phi: G \rightarrow \prod_{G_{n}} K_{n}$ as in Lemma 11.

\section{$3.3 \phi: G \rightarrow \Pi_{G_{n}} K_{n}$ is onto}

Lemma 14 Suppose $n \geq 1$ and $\left[k_{n}\right] \in K_{n}$. Then there exists $\beta \in\left[k_{n}\right]$ such that $\beta(s)(i, 0)=(i, 0)$ for all $s \in[0,1]$ and for all $i \leq n-1$.

Proof. Since $\left[k_{n}\right] \in i m\left(i_{n}^{*}\right)$, we may choose $\alpha \in\left[k_{n}\right]$ such that the $i t h$ strand of $\alpha$ is straight for all $i>n$. The braid $\alpha$ can deformed in $X_{n}$ to a braid $\Gamma$ so that the first $n-1$ strands of $\Gamma$ lie over the closed half plane $\left(\infty, n-\frac{1}{3}\right] \times R$ as follows. Recalling the map $R_{n}$ from the proof of Lemma 13. fix $t$ and translate $\alpha(t)\left(Z_{n}\right)$ leftward at constant speed by a total amount $R_{n-1}(\alpha(t))$. The braid $\gamma=P_{n-1}(\Gamma)$ is equivalent to the trivial braid in $X_{n-1}$ and the first $n-1$ strands of $\gamma$ agree with the first $n-1$ 
strands of $\Gamma$. Let $\gamma_{t}$ be a path of braids in $X_{n-1}$ taking $\gamma$ to $i d$. By Lemma 4 extend $\gamma_{t}$ to a level preserving isotopy $h_{t}: R^{2} \times[0,1] \rightarrow R^{2} \times[0,1]$ such that and $h_{1}=i d_{\mid R^{2} \times[0,1]}$ and $h_{t}$ is the identity on the top and bottom. Now consider the path of braids $\Gamma_{t}=h_{t} h_{0}^{-1}(\Gamma)$. Note $\Gamma_{0}=\Gamma$ and the first $n-1$ strands of $\Gamma_{1}$ are straight. Let $\beta=\Gamma_{1}$.

Corollary $15 \phi$ is an epimorphism.

Proof. Suppose $\left\{\left[k_{1}\right],\left[k_{2}\right], ..\right\} \in \Pi_{G_{n}} K_{i}$.

Choose by Lemma $14 \beta_{n} \in\left[k_{n}\right]$ such that $\beta_{n}(s)(i, 0)=(i, 0)$ for $i \leq n-1$. Define $g:[1, \infty] \rightarrow X$ as

$$
g(n+t)=\left\{\begin{array}{cc}
\beta_{n}(t) & n \geq 1 \text { and } t \in[0,1] \\
\iota d & t=\infty
\end{array} .\right.
$$

Note $g$ is well defined and continuous when $t<\infty$ since $\beta_{n}(0)=$ $\beta_{n}(1)=i d$ for $n \geq 1$. Suppose $t=\infty, T_{n} \rightarrow \infty$ and $F \subset Z^{+}$is compact. Let $M=\max \{i \mid(i, 0) \in F\}$. For $T \geq M+1$ and $(i, 0) \in F$, $g(T)(i, 0)=(i, 0)$. Hence $g$ is continuous. Let $h:[0,1] \rightarrow[1, \infty]$ be any order preserving homeomorphism. Let $f=g h$. Note $f(0)=f(1)=$ $i d$. Note $\phi_{n}([f])=\left[\beta_{1} \beta_{2} \ldots \beta_{n}\right]$. Thus, recalling Lemma [11, $\left(\phi([f])_{n}=\right.$ $\phi_{n-1}([f])^{-1} \phi_{n}([f])=\left[\beta_{1} . . \beta_{n-1}\right]^{-1}\left[\beta_{1} \beta_{2} \ldots \beta_{n}\right]=\left[\beta_{n}\right]$. Hence $\phi$ is an epimorphism.

\section{4 $\phi: G \rightarrow \Pi_{G} K_{n}$ is one to one}

Lemma [16] says the following: Given an infinite pure braid $\alpha$, if the infinite braid $P_{n+1}(\alpha)$ is equivalent to the trivial braid on infinitely many strands, then $\alpha_{\mid Z_{n+1}}$ is equivalent to the trivial braid on $n+1$ strands.

Lemma 16 Suppose $[\alpha] \in \operatorname{ker} \phi_{n+1}$. Define $\beta:[0,1] \rightarrow I\left(Z_{n+1}, R^{2}\right)$ as $\beta(s)=\alpha(s)_{\mid Z_{n+1}}$. Then $[\beta]=[i d] \in \pi_{1}\left(I\left(Z_{n+1}, R^{2}\right), i d\right)$.

Proof. For $(s, t) \in[0,1] \times[0,1]$ define $\beta_{s}^{t}: Z_{n+1} \hookrightarrow R^{2}$ via

$\beta^{t}(s)(i, 0)=\left(T\left(t R_{n+1}(\alpha(s))\right)\right) \alpha(i, 0)$. Note $\beta^{0}=\beta, P_{n+1}(\alpha)_{\mid Z_{n+1}}=$ $\beta^{1}$ and $\beta^{1} \in[\beta] \in \pi_{1}\left(I\left(Z_{n+1}, R^{2}\right), i d\right)$. Since $\left[P_{n+1}(\alpha)\right]=[i d] \in \pi_{1}\left(X_{n}, i d\right)$ there exists $\gamma:[0,1] \rightarrow X_{n}$ such that $\gamma(0)=P_{n+1}(\alpha)$ and $\gamma(1)=i d$. Now observe $\gamma(t)_{\mid Z_{n+1}}$ connects $\beta^{1}$ to $i d$ within $[\beta] \in \pi_{1}\left(I\left(Z_{n+1}, R^{2}\right), i d\right)$.

Lemma 17 says the following. Suppose the pure braid on $n+1$ strands $\beta^{0}$ can be deformed to the trivial braid on $n+1$ strands, and $n$ strands of $\beta^{0}$ are straight. Then the deformation can be chosen to leave these $n$ strands invariant.

Lemma 17 Suppose $\left[\beta^{0}\right]=[i d] \in \pi_{1}\left(I\left(Z_{n+1}, R^{2}\right), i d\right)$ and for all $s \in$ $[0,1] \beta^{0}(s)_{Z_{n}}=i d_{Z_{n}}$. Then there exists $\gamma:[0,1] \rightarrow\left[\beta^{0}\right]$ such that $\gamma(0)=$ $\beta^{0}, \gamma(1)=i d$ and $\gamma(t)_{Z_{n}}=i d_{Z_{n}}$. 
Proof. Let $f:[0,1] \times[0,1] \rightarrow I\left(Z_{n+1}, R^{2}\right)$ satisfy $f(0, s)=\beta^{0}(s)$ and $f(1, s)=f(0, t)=f(1, t)=i d$. Let $q(s, t)=f(s, t)_{Z_{n}}$. Note for $(s, t) \in \partial([0,1] \times[0,1]) q(s, t)_{Z_{n}}=i d_{Z_{n}}$. Since $I\left(Z_{n}, R^{2}\right)$ is aspherical (Theorem 2[7]), viewed as a map of $S^{2}$ into $I\left(Z_{n}, R^{2}\right), q$ is inessential. By Proposition 3 the restriction map $\pi: H\left(R^{2}, R^{2}\right) \rightarrow I\left(Z_{n}, R^{2}\right)$ defined as $\pi(h)=h_{Z_{n}}$ determines a fibre bundle. Hence there exists a 'lift' $Q:[0,1] \times[0,1] \rightarrow H\left(R^{2}, R^{2}\right)$ such that $Q(s, t)=I D_{R^{2}}$ for $(s, t) \in$ $\partial([0,1] \times[0,1])$ and $\pi(Q)=q$. Let $\gamma(t)(s)=(Q(s, t))^{-1} f(s, t)$.

Lemma 18 establishes the inductive step of our proof that $\phi$ is one to one.

Lemma 18 Suppose $\left[\alpha_{0}\right] \in \operatorname{ker} \phi_{n+1}$ and for all $s \in[0,1] \alpha_{0}(s)_{\mid Z_{n}}=$ $i d_{Z_{n}}$. Then there exists a map $\Gamma:[0,1] \rightarrow\left[\alpha_{0}\right]$ such that $\Gamma(0)=\alpha_{0}$, $\Gamma(t)(s)_{\mid Z_{n}}=i d_{\mid Z_{n}}$ and $\Gamma(1)(s)_{\mid Z_{n+1}}=i d_{\mid Z_{n+1}}$.

Proof. Define $\beta_{0}:[0,1] \rightarrow I\left(Z_{n+1}, R^{2}\right)$ via $\beta_{0}(s)=\alpha_{0}(s)$ applying Lemmas 16] and 17let $\beta_{t}$ be a path homotopy from $\beta_{0}$ to $i d$ in $I\left(Z_{n+1}, R^{2}\right)$ such that the first $n$ strands of $\beta_{t}$ are straight. By Lemma 4 extend $\beta_{t}$ to a level preserving isotopy $h_{t}: R^{2} \times[0,1] \rightarrow R^{2} \times[0,1]$ such that $h_{1}=i d_{\mid R^{2} x[0,1]}$ and $h_{t}$ is the identity on the top and bottom level. Let $\Gamma_{t}=h_{t} h_{0}^{-1}\left(\alpha_{0}\right)$.

Lemma $19 \phi: G \rightarrow \prod_{G} K_{n}$ is one to one

Proof. By definition $\operatorname{ker}(\phi)=\cap_{i=n}^{\infty} \operatorname{ker}\left(\phi_{n}\right)$. To prove $\phi$ has trivial kernel we take $\left[g_{0}\right] \in \operatorname{ker} \phi$ and build a path $g_{t}$ (parametrized over $[0, \infty]$ ) from $g_{0}$ to $i d$ within $\left[g_{0}\right]$ by the following procedure. By induction assume we have defined the homotopy $g_{t}$ for $t \in[0, n]$ and that the first $n$ strands of $g_{n}$ are straight. Then straighten the $n+1$ strand of $g_{n}$ for $t \in[n, n+1]$ via methods established in Lemmas 16, 17, and 18, Define $g_{\infty}(s)=i d_{\mid Z^{+}}$. We must prove the function $q:[0, \infty] \rightarrow C\left([0,1], C\left(Z^{+}, R^{2}\right)\right)$ defined via $q(t)=g_{t}$ is continuous when $t=\infty$. By Proposition 2 it suffices to show that the following map $Q:[0, \infty] \times[0,1] \times Z^{+} \rightarrow R^{2}$ defined via $Q(t, s,(n, 0))=g_{t}(s)(n, 0)$ is continuous. Continuity is immediate if $t<\infty$. Suppose $t_{k} \rightarrow \infty$ and $s_{k} \rightarrow s$ and $\left(n_{k}, 0\right) \rightarrow(n, 0)$. Suppose $\varepsilon>0$. Choose $K$ such that if $k \geq K$ then $n_{k}=n$ and $t_{k}>n+1$. Suppose $k \geq K$. Then $Q\left(t_{k}, s_{k}, n_{k}\right)=(n, 0)=Q(\infty, s, n)$. Thus $q$ is continuous.

\section{5 $G$ is the inverse limit of the pure braid groups}

Theorem $20 \phi: G \rightarrow \Pi_{G_{n}} K_{n}$ is a homeomorphism and $G$ is topologically complete. 
Proof. To prove $\phi_{n}: G \rightarrow G_{n}$ is continuous apply Proposition [ 1 to the retraction $P_{n}: X \rightarrow X_{n}$. Thus the isomorphism $H^{*}: G \rightarrow \lim _{\leftarrow} G_{n}$ defined via $H^{*}([g])=\left(\phi_{1}([g]), \phi_{2}([g]), \ldots\right)$ is continuous. Note $\phi=\psi H^{*}$ where $\psi: \lim _{\leftarrow} G_{n} \rightarrow \prod_{G_{n}} K_{n}$ is the homeomorphism from Lemma 12. Thus $\phi$ is continuous. To check $\phi^{-1}$ is continuous it suffices, since $\Pi_{G_{n}} K_{n}$ is metrizable and $\phi^{-1}$ is a homomorphism, to show $\phi^{-1}$ preserves convergence at $([i d],[i d], \ldots)$. Suppose $\left(\left[k_{1}^{1}\right],\left[k_{2}^{1}\right], \ldots\right),\left(\left[k_{1}^{2}\right],\left[k_{2}^{2}\right], ..\right), \ldots \rightarrow$ $([i d],[i d], ..) \in \Pi_{G_{n}} K_{n}$. Choose $g^{i} \in \phi^{-1}\left(\left[k_{1}^{i}\right],\left[k_{2}^{i}\right], \ldots\right)$ such that ( considering $i m\left(g^{i}\right)$ as a subspace of $\left.R^{2} \times[0,1]\right)$ a maximal number of initial strands of $i m(g)$ are straight line segments. It follows that $g^{i} \rightarrow i d^{*}$ in $C([0,1], X)$ where $i d^{*}(t)=i d_{Z^{+}}$.

Hence $\left[g^{i}\right] \rightarrow\left[i d^{*}\right] \in G$ and thus $\phi^{-1}$ is continuous. Moreover $G$ is completely metrizable since $G$ is homeomorphic to the countable product of complete (discrete) spaces $K_{n}$.

\subsection{The complete braid group $\overline{B_{\infty}}$}

Recalling Theorem $\square$ let $\overline{B_{\infty}}=G\left(X, H\left(Z^{+}, Z^{+}\right)\right)$.

In the definition of $G\left(X, H\left(Z^{+}, Z^{+}\right)\right)$, when defining $K$, for convenience replace $[0,1]$ with $[0, \infty]$ the one point compactification of $[0, \infty)$.

Elements of $\overline{B_{\infty}}$ are path components of $K$, and the graph of each element of $K$ can be seen as a collection of arcs in $R^{2} \times[0, \infty]$ connecting $Z^{+} \times\{0\}$ to $Z^{+} \times\{\infty\}$. Roughly speaking, two such graphs are equivalent if one can be deformed into the other while leaving the endpoints fixed.

We show $\overline{B_{\infty}}$ is topologically complete by exhibiting a homeomorphism onto the product $G \times H\left(Z^{+}, Z^{+}\right)$.

Lemma 21 Define $\Pi: \overline{B_{\infty}} \rightarrow H\left(Z^{+}, Z^{+}\right)$such that $\Pi([b])(i)=b(\infty)(i)$. Suppose there exists a map $\sigma: H\left(Z^{+}, Z^{+}\right) \rightarrow \overline{B_{\infty}}$ such that $\Pi \sigma=i d$. Then $\overline{B_{\infty}}$ is homeomorphic to the product $G\left(X, i d_{Z^{+}}\right) \times H\left(Z^{+}, Z^{+}\right)$.

Proof. Define $T: K \rightarrow H\left(Z^{+}, Z^{+}\right)$via $T(k)=k(\infty)$. Note all path components of $H\left(Z^{+}, Z^{+}\right)$are trivial and thus $T$ induces the map $\Pi$ by Proposition 1 . Moreover $\Pi$ is a homomorphism and ker $\Pi$ is precisely $G\left(X, i d_{Z^{+}}\right)$, the fundamental group of $X$ based at $i d_{Z^{+}}$. Now consider the map $F: \operatorname{ker}(\Pi) \times H\left(Z^{+}, Z^{+}\right) \rightarrow \overline{B_{\infty}}$ defined via $F(g, \tau)=g * \sigma(\tau)$. Consider the map $P: \overline{B_{\infty}} \rightarrow \operatorname{ker}(\Pi) \times H\left(Z^{+}, Z^{+}\right)$defined via $P(b)=$ $\left(b *(\sigma \Pi b)^{-1}, \Pi b\right)$.

To check that $b *(\sigma \Pi b)^{-1} \in \operatorname{ker}(\Pi)$ note $\Pi\left(b *(\sigma \Pi b)^{-1}\right)=\Pi(b) *$ $\Pi\left((\sigma \Pi b)^{-1}\right)=\Pi(b) *[\Pi(\sigma \Pi b)]^{-1}=\Pi(b) *[\Pi b]^{-1}=i d$. Finally note $F P=i d$. Thus each of $F$ and $P$ is a homeomorphism.

Let $Z=\{1,2,3, \ldots\}$ with the discrete topology. We will show that $H(Z, Z)$ is a closed subspace of $E(Z, Z) \subset C(Z, Z)$, that $E(Z, Z)$ is a 
$G_{\delta}$ subspace of $C(Z, Z)$, and that $C(Z, Z)$ admits a complete metric. It follows that $H(Z, Z)$ admits a complete metric.

We now construct a 'normal form' for each permutation $\tau \in H(Z, Z)$ as an 'infinite word' $\sigma_{1 m_{1}} \sigma_{2 m_{2}} \ldots$ where $\sigma_{n m} \in \sigma_{\infty}$ has compact support.

For $1 \leq n \leq m \in Z^{+}$define $\sigma_{n m}: Z^{+} \rightarrow Z^{+}$via

$$
\sigma_{n m}(i)=\left\{\begin{array}{cc}
i+1 & n \leq i \leq m-1 \\
n & i=m \\
i & \text { otherwise }
\end{array}\right.
$$

Define $b_{n m}:[0,1] \rightarrow X$ such that $b_{n m}$ is continuous and

$$
\left\{\begin{array}{cc}
b_{n m}(1)(i, 0)=\sigma_{n m}(i) & \text { for all } i \\
b_{n m}(0)(i, 0)=(i, 0) & \text { for all } i \\
n-1<\left|b_{n m}(t)(i, 0)\right|<m+1 & n \leq i \leq m \text { and } n<m \\
b_{n m}(t)(i, 0)=(i, 0) & \text { otherwise }
\end{array}\right.
$$

Lemma 22 For each $\tau \in H(Z, Z)$ there exists a sequence

$$
\sigma_{1 m_{1}}, \sigma_{2 m_{2}}, . . \in H(Z, Z)
$$

such that for all $k \geq 1$

$$
\left(\tau^{-1}\right)_{\mid\{1, \ldots k\}}=\left(\sigma_{k m_{k}} \ldots \sigma_{2 m_{2}} \sigma_{1 m_{1}}\right)_{\mid\{1, . . k\}}^{-1} .
$$

Proof. Suppose $\tau \in H(Z, Z)$. Let $m_{1}=\tau^{-1}(1)$. Proceeding by induction suppose

$$
\left(\tau^{-1}\right)_{\mid\{1, \ldots k\}}=\left(\sigma_{k m_{k}} \ldots \sigma_{2 m_{2}} \sigma_{1 m_{1}}\right)_{\mid\{1, . . k\}}^{-1}
$$

Define $m_{k+1}=\sigma_{k m_{k}} \ldots \sigma_{2 m_{2}} \sigma_{1 m_{1}} \tau^{-1}(k+1)$. Since $\sigma_{k m_{k}} \ldots \sigma_{2 m_{2}} \sigma_{1 m_{1}}$ maps $\tau^{-1}\{1, ., k\}$ onto $\{1, . . k\}$ it follows that $m_{k+1} \geq k+1$. By the induction hypothesis if $i \leq k$ then $\left(\sigma_{k+1 m_{k+1}} \sigma_{k m_{k}} \ldots \sigma_{2 m_{2}} \sigma_{1 m_{1}}\right)^{-1}(i)=$ $\sigma_{1 m_{1}}^{-1} . . \sigma_{k m_{k}}^{-1}(i)=\tau^{-1}(i)$ since $\sigma_{k+1 m_{k+1}}$ fixes $\{1, . . k\}$ pointwise. If $i=k+1$ then

$$
\begin{gathered}
\left(\sigma_{k+1 m_{k+1}} \sigma_{k m_{k}} \ldots \sigma_{2 m_{2}} \sigma_{1 m_{1}}\right)^{-1}(k+1)= \\
\sigma_{1 m_{1}}^{-1} . . \sigma_{k m_{k}}^{-1} \sigma_{k+1 m_{k+1}}^{-1}(k+1)=\sigma_{1 m_{1}}^{-1} . \sigma_{k m_{k}}^{-1}\left(m_{k+1}\right)= \\
\sigma_{1 m_{1}}^{-1} . . \sigma_{k m_{k}}^{-1}\left(\sigma_{k m_{k}} \ldots \sigma_{2 m_{2}} \sigma_{1 m_{1}} \tau^{-1}(k+1)\right)=\tau^{-1}(k+1) .
\end{gathered}
$$

Lemma 23 There exists a continuous function $\sigma: H(Z, Z) \rightarrow \overline{B_{\infty}}$ such that $\sigma(\tau)(1)(i, 0)=(\tau(i), 0)$ for all $i \in H(Z, Z)$. 
Proof. Define $\sigma: H(Z, Z) \rightarrow K \subset C([0, \infty], X)$ as follows.

Given $\tau \in H(Z, Z)$ choose $\sigma_{1 m_{1}}, \sigma_{2 m_{2}},$. via Lemma 22 so that for all $k \geq 1\left(\tau^{-1}\right)_{\mid\{1, \ldots k\}}=\left(\sigma_{k m_{k}} \ldots \sigma_{2 m_{2}} \sigma_{1 m_{1}}\right)_{\mid\{1, . . k\}}^{-1}$. Define $\alpha_{\tau}:[0, \infty] \rightarrow X$ such that

$$
\alpha_{\tau}(t)(i, 0)=\left\{\begin{array}{cc}
(i, 0) & t=0 \\
b_{k m_{k}}(t) \alpha_{\tau}(k-1)(i, 0) & t \in(k-1, k] \\
(\tau(i), 0) & t=\infty
\end{array}\right.
$$

Note $\alpha_{\tau}$ is continuous at $k$ since $b_{k m_{k}}(0)=i d$. To check continuity at $t=\infty$. Suppose $(i, 0) \in Z^{+}$. Let $(n, 0)=(\tau(i), 0)$. Suppose $t \in(k, k+1]$ and $n+1 \leq k$. Then $\alpha_{\tau}(k+t)(i, 0)=b_{k m_{k}}(t) b_{k-1 m_{k-1}} \ldots b_{1 m_{1}}(1)(i, 0)=$

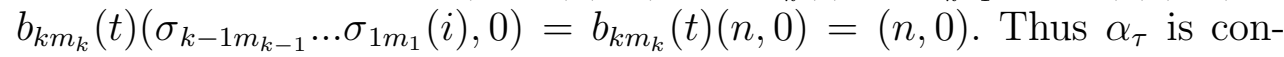
tinuous on $[0, \infty]$.

To check $\sigma$ is continuous suppose $\tau_{m} \rightarrow \tau \in H(Z, Z)$.

Choose $\sigma_{1 m_{1}}, \sigma_{2 m_{2}}, .$. via Lemma 22 so that for all $k \geq 1\left(\tau^{-1}\right)_{\mid\{1, \ldots k\}}=$ $\left(\sigma_{k m_{k}} \ldots \sigma_{2 m_{2}} \sigma_{1 m_{1}}\right)_{\mid\{1, . . k\}}^{-1}$. Suppose $(i, 0) \in Z^{+}$. Let $n=\tau(i)$. Choose $M>$ $n$ so that if $m \geq M$ then $\sigma_{k m_{k}}^{m}=\sigma_{k m_{k}}$ where $\sigma_{1 m_{1}}^{m}, \sigma_{2 m_{2}}^{m} \ldots$ is chosen for $\tau_{m}$ as in Lemma 22. Suppose $m \geq M$ and $t \in[0, \infty]$. Note $\alpha_{\tau_{m}}(t)(i, 0)=$ $\alpha_{\tau}(t)(i, 0)$. Thus $\sigma^{\wedge}$ is continuous.

Appealing to Proposition 1] let $\sigma: \Sigma_{\infty} \rightarrow \overline{B_{\infty}}$ be the induced map defined via $\sigma(\tau)=[\hat{\sigma}(\tau)]$.

Theorem $24 \overline{B_{\infty}}$ is topologically complete.

Proof. Applying Lemmas [21, [5] 23] and Theorem 20] we obtain a homeomorphism between $\overline{B_{\infty}}$ and the product of topologically complete spaces $G\left(X, i d_{Z^{+}}\right) \times H\left(Z^{+}, Z^{+}\right)$.

\subsection{A geometric description of convergence in $\overline{B_{\infty}}$.}

We indicate why convergence $b_{n} \rightarrow i d \in \overline{B_{\infty}}$ is characterized by the statement 'For each $M$ there exists $N$ such that if $n \geq N$ then the first $M$ strands of $b_{n}$ are trivial'. Recalling the proof of Lemma 21 note $b_{n} \rightarrow i d$ iff $\Pi\left(b_{n}\right) \rightarrow i d \in H\left(Z^{+}, Z^{+}\right)$and $b_{n} *\left(\sigma \Pi b_{n}\right)^{-1} \rightarrow i d \in G$. In $H\left(Z^{+}, Z^{+}\right)$convergence is pointwise. Thus $\Pi\left(b_{n}\right) \rightarrow i d$ iff for each $M$ there exists $N$ such that $n \geq N \Pi\left(b_{n}\right)(i)=i$ for $i \leq M$ iff (recalling the definition of $\sigma$ from Lemma 23) for each $M$ there exists $N$ such that the first $M$ strands of $\sigma\left(\Pi b_{n}\right)$ are trivial. In $G$, convergence to $i d$ is determined by pointwise convergence to $i d$ of the coordinates in $\Pi_{G_{n}} K_{n}$ as shown in Theorem 20, 


\subsection{There exist wild braids in $\overline{B_{\infty}}$}

Elements of the finite braid groups $B_{n}$ can be understood as path homotopy classes of homeomorphisms of the plane which start at the identity, and place the finite set $F_{n}$ onto itself at the last moment of the isotopy. However $\overline{B_{\infty}}$ cannot be understood in corresponding fashion due to the existence of 'wild' braids.

By definition elements of the pure braid subgroup $G \subset \overline{B_{\infty}}$ can be understood as equivalence classes of paths of embeddings of $Z^{+}$into the plane which start and stop at $i d_{Z^{+}}$. The set $Z^{+}$is allowed to move off itself in midjourney. Consider for $t \in[0,1]$ the following isotopy $g_{t}$ of $Z^{+}$. For $t \in\left[1-\frac{1}{n}, 1-\frac{1}{n+1}\right]$ let $(n, 0)$ perform a small clockwise orbit once around $(n+1,0)$ while leaving all other points of $Z^{+}$fixed. Let $S$ denote a simple closed curve bounding a convex disk $D$ such that $\{(1,0),(2,0)\} \subset \operatorname{int}(D)$ and $\emptyset=D \cap\{(3,0),(4,0), \ldots\}$. For $t \in[0,1)$ if we extend the isotopy $g_{t}$ to include $S$ we notice the diameter of $S$ is forcibly stretched by arbitrarily large amounts. Hence the isotopy cannot be extended to $S$ for $t \in[0,1]$ and hence the braid determined by $g_{t}$ is not determined by an ambient isotopy of the plane.

\section{References}

[1] Biss, Daniel K., The topological fundamental group and generalized covering spaces. Topology Appl. 124 (2002), no. 3, 355-371.

[2] Broto, K.; Vershinin, V. V., On the generalized homology of Artin groups.(Russian) Zap. Nauchn. Sem. S.-Peterburg. Otdel. Mat. Inst. Steklov. (POMI) 266 (2000), Teor. Predst. Din. Sist. Komb. i Algoritm. Metody. 5, 7-12, 336.

[3] Dehornoy, Patrick, Braid groups and left distributive operations. Trans. Amer. Math. Soc. 345 (1994), no. 1, 115-150.

[4] Dehornoy, Patrick, Transfinite braids and left distributive operations. Math. Z. 228 (1998), no. 3, 405-433.

[5] Dynnikov, I. A., Finitely presented groups and semigroups in knot theory. (Russian) Tr. Mat. Inst. Steklova 231 (2000), Din. Sist., Avtom. i Beskon. Gruppy, 231-248; translation in Proc. Steklov Inst. Math. 2000, no. 4 (231), 220-237.

[6] Fabel, Paul, The Mapping class group of a disk with infinitely many holes. Preprint http://front.math.ucdavis.edu/math.GT/0303042

[7] Fadell, Edward; Van Buskirk, James, On the braid groups of $E^{2}$ and $S^{2}$. Bull. Amer. Math. Soc. 671961 211-213.

[8] Moran, Siegfried, Cable knots and infinite necklaces of knots. Bull. Austral. Math. Soc. 51 (1995), no. 1, 17-31.

[9] Munkres, James R., Topology: a first course. Prentice-Hall, Inc., 
Englewood Cliffs, N.J., 1975.

[10] Kim, Djun Maximilian; Rolfsen, Dale, An ordering for groups of pure braids and fibre-type hyperplane arrangements. Canad. J. Math. 55 (2003), no. 4, 822-838.

[11] Vershinin, V. V., Braid groups and loop spaces. (Russian) Uspekhi Mat. Nauk 54 (1999), no. 2(326), 3-84; translation in Russian Math.Section 